\title{
Prospective Study of Infrared-Guided Patient Setup for Fractionated Thoracic Radiation
}

\author{
Aileen B. Chen'1, Yulia Lyatskaya', Joseph H. Killoran'1, Michelle Boyd"1, Shrooti Singh1, \\ Scott Kaplin², Aaron M. Allen² \\ ${ }^{1}$ Department of Radiation Oncology, Dana-Farber Cancer Institute/Brigham and Women's Hospital, Boston, MA, USA \\ ${ }^{2}$ Department of Radiation Oncology, Davidoff Center, Petach Tikvah, Israel \\ Email: ahron.alon@gmail.com
}

How to cite this paper: Chen, A.B., Lyatskaya, Y., Killoran, J.H., Boyd, M., Singh, S., Kaplin, S. and Allen, A.M. (2017) Prospective Study of Infrared-Guided Patient Setup for Fractionated Thoracic Radiation. International Journal of Medical Physics, Clinical Engineering and Radiation Oncology, 6, 313-322.

https://doi.org/10.4236/ijmpcero.2017.63028

Received: April 6, 2017

Accepted: August 20, 2017

Published: August 23, 2017

Copyright (c) 2017 by authors and Scientific Research Publishing Inc. This work is licensed under the Creative Commons Attribution International License (CC BY 4.0).

http://creativecommons.org/licenses/by/4.0/ (c) (i) Open Access

\begin{abstract}
Objective: The standard practice in fractionated radiation of using of visual alignment of tattoos with weekly portal imaging for radiation setup can miss setup errors. We previously reported on the feasibility of using an automated infrared-guided positioning system (iGPS) for daily radiation setup. In this study, we prospectively evaluate whether use of iGPS can improve daily setup errors for patients with thoracic malignancies. Methods: Multiple external infrared markers were placed on patients undergoing thoracic radiation at the time of simulation. Patients were immobilized using vacuum immobilization bag and wing-board. Patients were aligned for treatment using only the iGPS system. Daily portal images were then taken, and shifts in patient position were recorded. Differences between isocenter position using iGPS versus daily portal imaging were calculated. Results: Data were collected for 698 treatment sessions for 27 patients. We found that in $94.0 \%, 96.4 \%$, and $93.7 \%$ of treatment sessions, isocenter position differed between iGPS and daily portal imaging by $\leq 5 \mathrm{~mm}$ in the left/right(L/R), anterior/posterior(A/P), and superior/inferior(S/I) directions, respectively. Isocenter position differed by $5-10$ $\mathrm{mm}$ in $5.7 \%(\mathrm{~L} / \mathrm{R}), 3.6 \%(\mathrm{~A} / \mathrm{P})$, and $5.9 \%(\mathrm{~S} / \mathrm{I})$ of treatment sessions, and by $>10 \mathrm{~mm}$ in $0.3 \%(\mathrm{~L} / \mathrm{R}), 0.0 \%(\mathrm{~A} / \mathrm{P})$, and $0.4 \%(\mathrm{~S} / \mathrm{I})$ of treatment sessions. Three-dimensional shifts were also calculated, with differences in isocenter position as follows: $94.9 \% \leq 5 \mathrm{~mm}, 5.1 \% 5-10 \mathrm{~mm}$, and $0.2 \%>10 \mathrm{~mm}$. This compares favorably to our prior studies. Discussion: Daily treatment setup using an infrared-guided patient positioning system, correlates well with daily portal imaging and may help to improve daily treatment setup for patients with thoracic malignancies.
\end{abstract}

\section{Keywords}

Radiotherapy, Infrared Markers, Thoracic Malignancies 


\section{Introduction}

Accurate daily patient setup is crucial for minimizing the planning target volume expansion necessary for treatment of thoracic cancers. Prior work in lung cancer has predominantly focused on reducing or accounting for tumor motion [1] [2] [3] [4] [5]. However, several studies have also suggested that setup error can have a major contribution in determining treatment accuracy [4]. Traditionally, radiation oncologists have used visual alignment of skin tattoos to light fields, as well as weekly portal image verification [6] [7] [8]. More recent technologies have introduced the use of daily $\mathrm{kV}$ imaging, conebeam $\mathrm{CT}$, as well as surface-based systems to improve accuracy of daily setup [9] [10] [11], though all of the approaches have their inherent limitations.

In a prior study, we retrospectively evaluated the use of a surface-based infrared-guided patient positioning system (iGPS) for daily setup of thoracic patients and found that daily iGPS-based shifts corresponded well with shifts based on daily portal imaging. In addition, we observed that the iGPS might offer additional nonlinear information that could complement patient setup based on daily portal imaging [11]. Infrared-guided systems prove a simple, noninvasive means to evaluate patient setup [12] [13] [14] [15], though their uses for thoracic malignancies have thus far mostly been limited to breathing phase determination, rather than as a comprehensive setup tool. In summary, although infrared systems have shown promise their clinical applicability has been limited.

In this study, we report on the results of a prospective protocol evaluating the use of an in-house, automated, iGPS for primary daily setup of patients with thoracic malignancies [16].

\section{Methods and Materials}

\subsection{Study Cohort}

A total of 35 patients planned for fractionated, conformal thoracic radiation were prospectively enrolled on an institutional review board-approved protocol at the Dana-Farber Cancer Institute/Brigham and Women's Hospital Department of Radiation Oncology to evaluate iGPS as a tool for setup verification in patients with thoracic malignancies. To be eligible, patients had to be scheduled for radiation therapy with definitive intent and have an expected treatment duration of at least five weeks, with ECOG performance status less than two.

\subsection{Study Design}

The iGPS has been previously described in detail [16]. In brief, this system is based on the principle of using IR light to monitor highly reflective fiducial markers placed on the patient's skin, similar to other commercially available systems [12] [13] [14]. An example of a treatment room with the iGPS installed is shown in Figure 1(a). In addition, the iGPS has the unique capacity to collect information from multiple IR markers and comprehensively analyze the patient's position relative to the actual desired location for the number of markers 


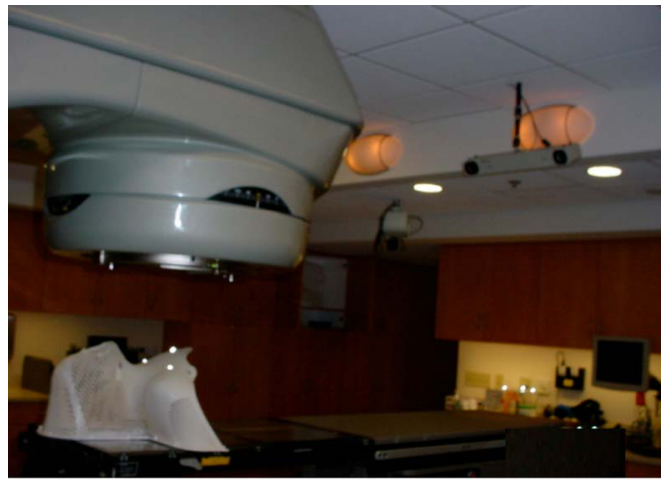

(a)

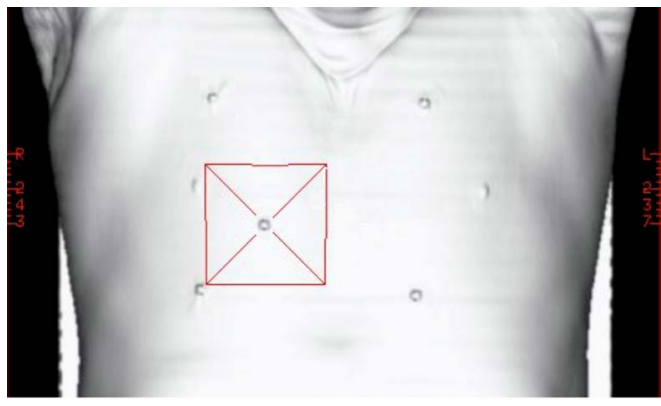

(b)

Figure 1. Schematic diagram showing the iGPS system (a) and placement of IR markers (b). (a) A picture of iGPS installed in treatment room. A mask with attached IR-markers illustrates position of the patient with respect to IR-camera; (b) A diagram showing position of IR-markers on a patient. The center of a red square illustrates CAX of the AP treatment beam.

selected. As described in previous studies, the use of multiple fiducial markers has been shown to provide both increased consistency and accuracy compared to the use of a single marker. Single fiducial markers can often lead to variation due to organ deformation and fiducial migration during treatment, while multiple markers provide a better representation of the desired organ [17]. Infrared markers were affixed to the patient's skin at the time of CT simulation in the pattern shown in Figure 1(b).

Four markers were placed at the corners of a rectangle on the patient's skin, two on the upper chest wall at the mid-axillary line, and two inferiorly at the costal border at the mid-axillary line. One marker, termed the central marker, was placed on the central axis of the AP beam.

At the time of simulation, patients were immobilized with a wingboard and vacuum immobilization bag (Vac-loc bag, MedTec, Orange City, IO) for the upper torso. [18] The five infrared iGPS markers were affixed to the patient's skin at the time of computed tomography (CT) simulation, and the position and location of each infrared marker was located by iGPS within the simulator room. The position of each marker was then marked on the patient's skin with a tattoo and photographs documenting tattoo locations were taken.

At the time of treatment, the five infrared markers were again placed on the patient's skin, and their position was detected by iGPS within the treatment 
room. The patient was then positioned using iGPS only, by comparing the position of each iGPS marker to its expected position. Using numerical feedback from iGPS, radiation therapists shifted the patients to the proper location. Daily portal images were taken following setup by the iGPS.

\subsection{Data Collection}

The routine clinical practice at the time of the study was to use weekly portal image measurements for routine patient setup evaluation. We have previously shown in a retrospective analysis that daily iGPS shifts correlate well with shifts based on analysis of daily electronic portal imaging [11]. Therefore, in this study, patients were positioned based on iGPS coordinates only, and daily AP and lateral portal images were obtained for post-treatment analysis.

Following completion of therapy, all differences in isocenter position were independently reviewed and recorded by a single senior therapist (MB) to the nearest $\mathrm{mm}$, to minimize the effect of inter-user variability.

\subsection{Data Analysis}

Isocenter position, as determined by the daily portal images, was considered the gold standard, with all shifts from the iGPS determined isocenter position considered deviations. The results are reported as the percentage of treatment sessions when the iGPS determined isocenter position differed from the daily portal image isocenter position by $>10 \mathrm{~mm}$ and $>5 \mathrm{~mm}$. These shifts were felt to be clinically significant since a $5 \mathrm{~mm}$ margin for setup error is typically used at our institution. In addition, we analyzed these shifts according to the method described by van Herk to characterize the systematic and random errors [19].

Daily patient setup using iGPS was compared to daily portal imaging, as the gold standard. The study was limited to the evaluation of the setup only, and no internal target motion was addressed. For the days, on which port films were available and shifts recorded, the mean of the shifts and standard deviation was calculated in each direction $(\mathrm{A} / \mathrm{P}, \mathrm{L} / \mathrm{R}$, and $\mathrm{S} / \mathrm{I})$ for each individual patient. The average of the mean values was calculated to provide a measure of systematic error (M). The standard deviation of mean for individual patients was obtained as characteristic of standard deviation of systematic error $(\Sigma)$ and rms of the standard deviations was used as a measure of standard deviation of the random error $(\sigma)$, as suggested by van Herk. In addition, the number of days were calculated for which shifts were $<5 \mathrm{~mm}$, between $5 \mathrm{~mm}$ and $1 \mathrm{~cm}$ and greater than $1 \mathrm{~cm}$. This was done for each individual patient and the total. Percentages of days with shifts $<5 \mathrm{~mm}$, between $5 \mathrm{~mm}$ and $1 \mathrm{~cm}$ and $>1 \mathrm{~cm}$ were also calculated.

\section{Results}

Between April 2007 and September 2009 we enrolled 35 patients on protocol. Of these, 1 died, 1 withdrew consent, and 2 had progressive disease before radiation therapy could be started. An additional 4 patients were unable to have iGPS used during treatment due to technical issues with marker detection for superiorly- 
located tumors. A total of 27 patients treated during 830 treatment sessions with $698 \mathrm{AP}$ and 694 lateral portal images were available to be analyzed.

We found that in $94.0 \%, 96.4 \%$, and $93.7 \%$ of treatment sessions, isocenter position differed between iGPS and daily portal imaging by $\leq 5 \mathrm{~mm}$ in the left/ right $(\mathrm{L} / \mathrm{R})$, anterior/posterior $(\mathrm{A} / \mathrm{P})$, and superior/ inferior $(\mathrm{S} / \mathrm{I})$ directions, respectively. Isocenter position differed by $5-10 \mathrm{~mm}$ in $5.7 \%(\mathrm{~L} / \mathrm{R}), 3.6 \%(\mathrm{~A} / \mathrm{P})$, and $5.9 \%(\mathrm{~S} / \mathrm{I})$ of treatment sessions, and by $>10 \mathrm{~mm}$ in $0.3 \%(\mathrm{~L} / \mathrm{R}), 0.0 \%(\mathrm{~A} / \mathrm{P})$, and $0.4 \%(\mathrm{~S} / \mathrm{I})$ of treatment sessions. Three-dimensional shifts were also calculated, with differences in isocenter position as follows: $94.9 \% \leq 5 \mathrm{~mm}, 5.1 \% 5$ - 10 $\mathrm{mm}$, and $0.2 \%>10 \mathrm{~mm}$. Table 1 shows the overall frequency of port-film shifts found after iGPS-based setup for all images and treatment sessions.

Table 2 shows mean shifts from iGPS compared to port-films for each analyzed patient, as well as mean and standard deviation values. In addition, Table 3 shows the systematic error (M) and standard deviation for a systematic error $(\Sigma)$ and standard deviation for random error $(\sigma)$.

We did not observe a systematic discrepancy between iGPS and port-film based setup (mean $<1 \mathrm{~mm}$ in all directions). Random error for iGPS compared to port-film based setup was $<2 \mathrm{~mm}$ in all directions, based on standard deviation values. The standard deviation of the random error was $<5 \mathrm{~mm}$, with higher accuracy in anterior direction $(<2 \mathrm{~mm})$. We examined if tumor location or patient age would impact on the degree of setup error. Although the overall number of patients in the different categories was small we did not see improved setup for upper lobe isocenters vs. lower lobe isocenters. In addition, there was no observable difference in the results depending on the age of the patient. This data is summarized in Table 4 and Table 5.

\section{Discussion}

In this study, we have expanded on prior work demonstrating the feasibility of daily radiation setup using an infrared marker based positioning system, by reporting on results from a prospective protocol. The results from the current study compare favorably to our prior study, where we found that standard setup with tattoos, laser marking, and weekly portal imaging yielded daily setup errors of $0 \mathrm{~mm}$ in $18 \%, 0-5 \mathrm{~mm}$ in $42 \%, 5-10 \mathrm{~mm}$ in $30 \%$, and $>10 \mathrm{~mm}$ in $10 \%$ of patients, compared to daily portal imaging [11].

Although, the use of daily $\mathrm{kV}$ imaging for image-guided radiation setup has grown increasing common over the past few years, our study demonstrates that

Table 1. Frequency of port film shifts found after daily iGPS-based setup for 27 patients.

\begin{tabular}{ccccc}
\hline Shifts & Left/Right (\%) & Ant/Post (\%) & Sup/Inf (\%) & 3-D (\%) \\
\hline $0-5 \mathrm{~mm}$ & $656(94.0 \%)$ & $669(96.4 \%)$ & $650(93.7 \%)$ & $658(94.8 \%)$ \\
$5.1-10 \mathrm{~mm}$ & $40(5.7 \%)$ & $25(3.6 \%)$ & $41(5.9 \%)$ & $35(5.0 \%)$ \\
$>10 \mathrm{~mm}$ & $2(0.3 \%)$ & $0(0.0 \%)$ & $3(0.4) \%$ & $1(0.2 \%)$ \\
Totals & $698(100 \%)$ & $694(100 \%)$ & $694(100 \%)$ & $694(100 \%)$ \\
\hline
\end{tabular}


Table 2. Port-film shifts found after iGPS-based setup*.

\begin{tabular}{|c|c|c|c|c|c|c|}
\hline \multirow{2}{*}{$\mathrm{Pt} \#$} & \multicolumn{3}{|c|}{ Mean shifts (mm) } & \multicolumn{3}{|c|}{ Standard deviation (STD) } \\
\hline & Left & Ant & Sup & Left & Ant & Sup \\
\hline 1 & 0.4 & 0.4 & 1.2 & 1.7 & 1.4 & 4.8 \\
\hline 2 & 1.4 & 2.6 & -1.3 & 2.1 & 1.7 & 4.1 \\
\hline 3 & 4.5 & -2.5 & -0.9 & 3.0 & 2.7 & 1.6 \\
\hline 4 & 3.8 & -0.2 & 0.3 & 3.5 & 1.5 & 3.2 \\
\hline 5 & 1.5 & -0.7 & -0.1 & 1.5 & 1.5 & 0.5 \\
\hline 6 & -2.7 & -0.6 & -2.5 & 4.8 & 1.6 & 3.5 \\
\hline 7 & -1.6 & 0.0 & 1.8 & 2.9 & 0.9 & 2.3 \\
\hline 8 & 2.3 & -1.5 & -3.6 & 2.1 & 2.1 & 4.5 \\
\hline 9 & 1.9 & -1.6 & -1.1 & 2.9 & 2.6 & 4.5 \\
\hline 10 & -0.6 & -2.6 & -0.2 & 1.3 & 3.0 & 0.9 \\
\hline 11 & 3.9 & -4.2 & 2.0 & 4.7 & 3.7 & 3.1 \\
\hline 12 & -0.8 & -2.3 & -0.8 & 1.8 & 2.1 & 1.9 \\
\hline 13 & -1.0 & 1.5 & 4.8 & 3.5 & 2.5 & 4.0 \\
\hline 14 & 0.2 & -0.5 & -1.1 & 1.5 & 1.1 & 3.8 \\
\hline 15 & -0.9 & -2.6 & -1.7 & 2.0 & 3.6 & 2.6 \\
\hline 16 & 1.5 & -1.3 & -0.9 & 2.2 & 2.7 & 3.6 \\
\hline 17 & 0.4 & -2.4 & -0.8 & 1.0 & 2.0 & 1.5 \\
\hline 18 & 1.1 & -1.4 & 0.0 & 1.6 & 2.0 & 0.0 \\
\hline 19 & 0.2 & 0.4 & -0.8 & 0.7 & 1.2 & 2.8 \\
\hline 20 & 0.3 & -1.9 & -0.7 & 1.1 & 1.9 & 1.3 \\
\hline 21 & 0.5 & 0.2 & -0.1 & 1.1 & 1.1 & 1.7 \\
\hline 22 & 1.8 & 1.0 & -0.4 & 3.0 & 2.3 & 1.1 \\
\hline 23 & -0.1 & -0.1 & -0.4 & 0.7 & 0.6 & 1.9 \\
\hline 24 & 0.0 & -1.5 & 0.3 & 0.0 & 2.6 & 3.0 \\
\hline 25 & 0.0 & 0.3 & 0.3 & 1.8 & 2.3 & 1.1 \\
\hline 26 & -1.3 & -0.8 & -0.4 & 3.5 & 1.7 & 2.9 \\
\hline 27 & 0.7 & 0.1 & -0.5 & 1.6 & 1.1 & 1.2 \\
\hline Mean & 0.6 & -0.8 & -0.3 & 2.1 & 2.0 & 2.5 \\
\hline STD & 1.7 & 1.5 & 1.6 & 1.2 & 0.8 & 1.3 \\
\hline
\end{tabular}

Table 3. Statistical analysis of the shifts from Table 2. Here, $M$ is the group systematic error, $\Sigma$ is the standard deviation of systematic error, and $\sigma$ is the standard deviation of random error. All values are in $\mathrm{mm}$.

\begin{tabular}{cccc}
\hline & Left & Ant & Sup \\
\hline $\mathbf{M}(\mathrm{mm})$ & 0.6 & -0.8 & -0.3 \\
$\Sigma(\mathrm{mm})$ & 1.7 & 1.5 & 1.5 \\
$\sigma(\mathrm{mm})$ & 3.6 & 1.6 & 4.7 \\
\hline
\end{tabular}


Table 4. Port-film mean shifts based on tumor location.

\begin{tabular}{cccc}
\hline \multirow{2}{*}{ Tumor location } & \multicolumn{3}{c}{ Mean shifts (mm) } \\
\cline { 2 - 4 } & Left & Ant & Sup \\
\hline Right Upper Lobe (10) & 1.4 & 1.2 & 1.4 \\
Right Middle Lobe (2) & 0.8 & 1.6 & 0.6 \\
Right Lower Lobe (8) & 1.7 & 1.5 & 0.7 \\
Right Hilar (1) & 0 & 1.5 & 0.3 \\
Left Upper Lobe (5) & 1.4 & 1.1 & 1.3 \\
Left Lower Lobe (1) & 0 & 0.3 & 0.3 \\
\hline
\end{tabular}

Table 5. Port-film mean shifts based on age at start of study.

\begin{tabular}{cccc}
\hline \multirow{2}{*}{ Age (Years) } & \multicolumn{3}{c}{ Mean shifts (mm) } \\
\cline { 2 - 4 } & Left & Ant & Sup \\
\hline $40-49(1)$ & 2.7 & 0.6 & 2.5 \\
$50-59(6)$ & 1.5 & 1.7 & 0.6 \\
$60-69(9)$ & 1.2 & 1.1 & 0.8 \\
$70-79(7)$ & 1.1 & 1.0 & 1.6 \\
$80-89(4)$ & 0.9 & 1.5 & 0.9 \\
\hline
\end{tabular}

an automated surface marker-based system can also be effective and has the potential advantage of limiting excess radiation exposure from daily imaging, as well as reducing the time required by obtaining and evaluating daily portal images. Although, our study evaluates an in-house surface-based marker system, several surface-based systems have been developed commercially over the past several years for treatment setup [12]. Our study provides reassurance that these surface-based systems are a feasible alternative to $\mathrm{x}$-ray based systems and can be used prospectively for daily patient setup.

There are other products that use surface imaging to aid in daily setup. One of the most commonly utilized is a 3-D surface camera system, AlignRT, produced by Vision RT Ltd. (London, England). This system has shown utility in setup of breast cancer patients. It differs from iGPS in that it uses a region of interest (ROI) often adjacent to the region of treatment. Recent data showed use of AlignRT to reduce setup errors as but an average of $9 \mathrm{~mm}$ shifts remain when compared to portal imaging [20]. Another similar study of this technique showed mean displacements were $4.1 \pm 2.6 \mathrm{~mm}, 2.7 \pm 1.4 \mathrm{~mm}$, and $2.6 \pm 1.2 \mathrm{~mm}$ in the anteroposterior (AP), superoinferior (S/I), and left-right (L/R) directions, respectively [21]. In addition, several studies have been conducted to analyze the accuracy and efficiency of AlignRT for patients receiving deep inspiration breath-hold (DIBH) radiation therapy for left-sided breast cancer. Results proved that the DIBH patient setup with AlignRT provided improved accuracy and reduced mean treatment time required for each patient while providing adequate sparing of cardiac tissue [22] [23] [24]. Our system provided very sim- 
ilar results to this commercially available system when compared for thoracic radiotherapy setup. It would suggest that both a localized ROI and 5 displaced markers over standard positions are comparable to achieve accurate non invasive patient setups. Overall lesions in the upper thorax were more highly reproducible rather than lower lobe lesions as can be seen from Table 4 .

There are several limitations to our study. Although, 31 patients consented to iGPS analysis and were eligible for study, in 4 of these patients, our in-house system had difficulty detecting and recording marker location. This problem occurred in patients with very superiorly located tumors because our infrared cameras required a direct line of sight in order to detect marker location. This problem was alleviated with subsequent patients by carefully selecting and modifying marker location for patients with very superiorly located tumors. In addition, we used daily portal imaging as a gold standard for comparison. However, daily portal imaging does not necessarily reflect true target volume location, particularly when the target volume is displaced from bony anatomy or may shift during the course of treatment. Likewise, surface-based treatment setup does not necessarily reflect the location of the target volume. One advantage of the iGPS system is that, with the placement of several different markers and the use of an automated system, rotational shifts can be more easily detected. Similar results have been recently published which confirm these hypotheses [25]. Additional analysis, using 3D imaging, such as conebeam $\mathrm{CT}$, may help to further assess the accuracy of surface-based systems relative to internal target volumes.

\section{Conclusions}

Daily treatment setup using an infrared-guided patient positioning system, correlates well with daily portal imaging and may help to improve daily treatment setup for patients with thoracic malignancies. This technique and other similar approaches have the potential to achieve accurate daily setups without adding radiation dose associated with $\mathrm{KV}$ - or MV imaging.

This work was presented in part at the American Society of Radiation Oncology Annual Meeting 2010.

\section{Acknowledgements}

This project was funded by a Kaye Family Technology Grant.

\section{Conflicts of Interest}

No actual or potential conflicts of interest exist.

\section{References}

[1] Erridge, S.C., Seppenwoolde, Y., Muller, S.H., et al. (2003) Portal Imaging to Assess Set-Up Errors, Tumor Motion and Tumor Shrinkage during Conformal Radiotherapy of Non-Small Cell Lung Cancer. Radiotherapy and Oncology, 66, 75-85. https://doi.org/10.1016/S0167-8140(02)00287-6

[2] Seppenwoolde, Y., Shirato, H., Kitamura, K., et al. (2002) Precise and Real-Time 
Measurement of 3D Tumor Motion in Lung Due to Breathing and Heartbeat, Measured during Radiotherapy. International Journal of Radiation Oncology Biology Physics, 53, 822-834. https://doi.org/10.1016/S0360-3016(02)02803-1

[3] Cho, B.C., Van Herk, M., Mijnheer, B.J., et al. (2002) The Effect of Set-Up Uncertainties, Contour Changes, and Tissue Inhomogeneities on Target Dose-Volume Histograms. Medical Physics, 29, 2305-2318. https://doi.org/10.1118/1.1508800

[4] Engelsman, M., Damen, E.M., De Jaeger, K., et al. (2001) The Effect of Breathing and Set-Up Errors on the Cumulative Dose to a Lung Tumor. Radiotherapy and Oncology, 60, 95-105. https://doi.org/10.1016/S0167-8140(01)00349-8

[5] Schwarz, M., Van der Geer, J., Van Herk, M., et al. (2006) Impact of Geometrical Uncertainties On 3D CRT and IMRT Dose Distributions for Lung Cancer Treatment. International Journal of Radiation Oncology Biology Physics, 65, 1260-1269. https://doi.org/10.1016/j.ijrobp.2006.03.035

[6] Hurkmans, C.W., Remeijer, P., Lebesque, J.V., et al. (2001) Set-Up Verification Using Portal Imaging; Review of Current Clinical Practice. Radiotherapy and Oncology, 58, 105-120. https://doi.org/10.1016/S0167-8140(00)00260-7

[7] Kutcher, G.J., Coia, L., Gillin, M., et al. (1994) Comprehensive QA for Radiation Oncology: Report of AAPM Radiation Therapy Committee Task Group 40. Medical Physics, 21, 581-618. https://doi.org/10.1118/1.597316

[8] Rodrigus, P., Van den Weyngaert, D. and Van den Bogaert, W. (1987) The Value of Treatment Portal Films in Radiotherapy for Bronchial Carcinoma. Radiotherapy and Oncology, 9, 27-31. https://doi.org/10.1016/S0167-8140(87)80216-5

[9] Dawson, L.A. and Jaffray, D.A. (2007) Advances in Image-Guided Radiation Therapy. Journal of Clinical Oncology, 25, 938-946. https://doi.org/10.1200/JCO.2006.09.9515

[10] Brahme, A., Nyman, P. and Skatt, B. (2008) 4D Laser Camera for Accurate Patient Positioning, Collision Avoidance, Image Fusion and Adaptive Approaches during Diagnostic and Therapeutic Procedures. Medical Physics, 35, 1670-1681. https://doi.org/10.1118/1.2889720

[11] Lyatskaya, Y., James, S., Killoran, J.H., et al. (2008) Infrared-Guided Patient Setup for Lung Cancer Patients. International Journal of Radiation Oncology Biology Physics, 71, 1124-1133. https://doi.org/10.1016/j.ijrobp.2007.11.050

[12] Meeks, S.L., Tome, W.A., Willoughby, T.R., et al. (2005) Optically Guided Patient Positioning Techniques. Seminars in Radiation Oncology, 15, 192-201.

https://doi.org/10.1016/j.semradonc.2005.01.004

[13] Soete, G., Van de Steene, J., Verellen, D., et al. (2002) Initial Clinical Experience with Infrared-Reflecting Skin Markers in the Positioning of Patients Treated by Conformal Radiotherapy for Prostate Cancer. International Journal of Radiation Oncology Biology Physics, 52, 694-698. https://doi.org/10.1016/S0360-3016(01)02642-6

[14] Baroni, G., Ferrigno, G. and Pedotti, A. (1998) Implementation and Application of Real-Time Motion Analysis Based on Passive Markers. Medical \& Biological Engineering \& Computing, 36, 693-703. https://doi.org/10.1007/BF02518871

[15] Herfarth, K.K., Debus, J., Lohr, F., et al. (2000) Extracranial Stereotactic Radiation Therapy: Set-Up Accuracy of Patients Treated for Liver Metastases. International Journal of Radiation Oncology Biology Physics, 46, 329-335. https://doi.org/10.1016/S0360-3016(99)00413-7

[16] Lyatskaya, Y., Lu, H.M. and Chin, L. (2006) Performance and Characteristics of an IR Localizing System for Radiation Therapy. Journal of Applied Clinical Medical Physics, 7, 18-37. https://doi.org/10.1120/jacmp.v7i2.2190 
[17] Kudchadker, R.J., Lee, A.K., Yu, Z.H., et al. (2009) Effectiveness of Using Fewer Implanted Fiducial Markers for Prostate Target Alignment. International Journal of Radiation Oncology Biology Physics, 74, 1283-1289. https://doi.org/10.1016/j.ijrobp.2009.02.033

[18] Halperin, R., Roa, W., Field, M., et al. (1999) Setup Reproducibility in Radiation Therapy for Lung Cancer: A Comparison between T-Bar and Expanded Foam Immobilization Devices. International Journal of Radiation Oncology Biology Physics, 43, 211-216. https://doi.org/10.1016/S0360-3016(98)00354-X

[19] Van Herk, M. (2004) Errors and Margins in Radiotherapy. Seminars in Radiation Oncology, 14, 52-64. https://doi.org/10.1053/j.semradonc.2003.10.003

[20] Padilla, L., Kang, H., Washington, M., et al. (2014) Assessment of Interfractional Variation of the Breast Surface Following Conventional Patient Positioning for Whole-Breast Radiotherapy. Journal of Applied Clinical Medical Physics, 15, 4921. https://doi.org/10.1120/jacmp.v15i5.4921

[21] Shah, A.P., Dvorak, T., Curry, M.S., et al. (2013) Clinical Evaluation of Inter-fractional Variations for Whole Breast Radiotherapy Using 3-Dimensional Surface Imaging. Practical Radiation Oncology, 3, 16-25.

https://doi.org/10.1016/j.prro.2012.03.002

[22] Alderliesten, T., Sonke, J.J., Betgen, A., et al. (2013) Accuracy Evaluation of a 3Dimensional Surface Imaging System for Guidance in Deep-Inspiration BreathHold Radiation Therapy. International Journal of Radiation Oncology Biology Physics, 85, 536-542. https://doi.org/10.1016/j.ijrobp.2012.04.004

[23] Tang, X., Zagar, T.M., Bair, E., et al. (2014) Clinical Experience with 3-Dimensional Surface Matching-Based Deep Inspiration Breath Hold for Left-Sided Breast Cancer Radiation Therapy. Practical Radiation Oncology, 4, e151-e158. https://doi.org/10.1016/j.prro.2013.05.004

[24] Gierga, D.P., Turcotte, J.C., Sharp, G.C., et al. (2012) A Voluntary Breath-Hold Treatment Technique for the Left Breast with Unfavorable Cardiac Anatomy Using Surface Imaging. International Journal of Radiation Oncology Biology Physics, 84, e663-e668. https://doi.org/10.1016/j.ijrobp.2012.07.2379

[25] Smadi, M.P., Esmaili, T.A. and Nankali, S. (2016) Investigation of the Optimum Location of External Markers for Patient Setup Accuracy Enhancement at External Beam Radiotherapy. Journal of Applied Clinical Medical Physics, 17, 6265.

\section{Scientific Research Publishing}

Submit or recommend next manuscript to SCIRP and we will provide best service for you:

Accepting pre-submission inquiries through Email, Facebook, LinkedIn, Twitter, etc. A wide selection of journals (inclusive of 9 subjects, more than 200 journals)

Providing 24-hour high-quality service

User-friendly online submission system

Fair and swift peer-review system

Efficient typesetting and proofreading procedure

Display of the result of downloads and visits, as well as the number of cited articles

Maximum dissemination of your research work

Submit your manuscript at: http://papersubmission.scirp.org/

Or contact ijmpcero@scirp.org 